\title{
Fractional charge and quantized current in the quantum spin Hall state
}

\author{
XIAO-LIANG QI, TAYLOR L. HUGHES AND SHOU-CHENG ZHANG* \\ Department of Physics, McCullough Building, Stanford University, Stanford, California 94305-4045, USA \\ *e-mail: sczhang@stanford.edu
}

Published online: 16 March 2008; doi:10.1038/nphys913

Soon after the theoretical proposal of the intrinsic spin Hall effect $^{1,2}$ in doped semiconductors, the concept of a time-reversal invariant spin Hall insulator ${ }^{3}$ was introduced. In the extreme quantum limit, a quantum spin Hall (QSH) insulator state has been proposed for various systems ${ }^{4-6}$. Recently, the QSH effect has been theoretically proposed ${ }^{6}$ and experimentally observed ${ }^{7}$ in HgTe quantum wells. One central question, however, remains unanswered-what is the direct experimental manifestation of this topologically non-trivial state of matter? In the case of the quantum Hall effect, it is the quantization of the Hall conductance and the fractional charge of quasiparticles, which are results of non-trivial topological structure. Here, we predict that for the QSH state a magnetic domain wall induces a localized state with half the charge of an electron. We also show that a rotating magnetic field can induce a quantized d.c. electric current, and vice versa. Both of these physical phenomena are expected to be direct and experimentally observable consequences of the non-trivial topology of the QSH state.

The quantum spin Hall (QSH) insulators are time-reversal invariant and have a bulk charge-excitation gap. However, this system also possesses topologically protected gapless edge states that lie inside the bulk insulating gap. The edge states of the QSH insulator state differ from the quantum Hall effect and have a distinct helical property: two states with opposite spin polarization counterpropagate at a given edge $e^{4,8,9}$. The edge states come in Kramers's doublets, and time-reversal symmetry ensures the crossing of their energy levels at special points in the Brillouin zone. Because of this level crossing, the spectrum of a QSH insulator cannot be adiabatically deformed into a topologically trivial insulating state; therefore, in this precise sense, the QSH insulators represent a topologically distinct new state of matter.

The idea of fractional charge induced at a domain wall goes back to the Su-Schrieffer-Heeger model ${ }^{10}$. (Recently, this model has been applied to describe the fractional charge carried by 'edge solitons' or bulk quasiparticles of two-dimensional quantum Hall systems ${ }^{11}$.) For spinless fermions, a mass domain wall induces a localized state with one-half charge. However, for a real material such as polyacetylene, two spin orientations are present for each electron, and because of this doubling, a domain wall in polyacetylene only carries integer charge; the proposal of Su, Schrieffer and Heeger, and its counterpart in field theory, the Jackiw-Rebbi model ${ }^{12}$, have never been experimentally realized. Conventional one-dimensional electronic systems have four basic degrees of freedom, right- and left-movers with each spin orientation. However, a helical liquid at a given edge of the QSH insulator has only two: a spin-up (or -down) right-mover and a spin-down (or -up) left-mover. Therefore, the helical liquid has half the degrees of freedom of a conventional one-dimensional system, and thus avoids the doubling problem. Because of this fundamental topological property of the helical liquid, a domain wall carries charge $e / 2$. We propose a Coulomb blockade experiment to observe this fractional charge. As a temporal analogue of the fractional charge effect, the pumping of a quantized charge current during each periodic rotation of a magnetic field is also proposed. This provides a direct realization of Thouless's topological pumping ${ }^{13}$.

We can express the effective theory for the edge states of a nontrivial QSH insulator as

$$
H_{0}=v_{\mathrm{F}} \int \mathrm{d} x\left(\psi_{\mathrm{R}+}^{\dagger} i \partial_{x} \psi_{\mathrm{R}+}-\psi_{\mathrm{L}-}^{\dagger} i \partial_{x} \psi_{\mathrm{L}-}\right)=v_{\mathrm{F}} \int \mathrm{d} x \Psi^{\dagger} i \sigma^{3} \partial_{x} \Psi,
$$

where \pm indicate members of a Kramers's doublet, $\mathrm{L} / \mathrm{R}$ indicate left- or right-movers, $v_{\mathrm{F}}$ is the Fermi velocity and $\Psi=\left(\psi_{\mathrm{R}+}, \psi_{\mathrm{L}-}\right)^{T}$ (refs 8,9 ). These helical fermion states have only two degrees of freedom; the spin polarization is correlated with the direction of motion.

A mass term, being proportional to the Pauli matrices $\left(\sigma_{1,2,3}\right)$, can only be introduced in the hamiltonian by coupling to a T-breaking external field such as a magnetic field or aligned magnetic impurities. To leading order in perturbation theory, a magnetic field generates the mass terms

$$
H_{\mathrm{M}}=\int \mathrm{d} x \Psi^{\dagger} \sum_{a=1,2,3} m_{a}(x, t) \sigma_{a} \Psi=\int \mathrm{d} x \Psi^{\dagger} \sum_{a, i} t_{a i} B_{i}(x, t) \sigma_{a} \Psi,
$$

where $B_{i}$ is the three-dimensional magnetic field and the model-dependent coefficient matrix $t_{a i}$ is determined by the coupling of the edge states to the magnetic field. According to the work of Goldstone and Wilczek ${ }^{14}$, at zero temperature the ground-state charge density and current in a background field $m_{a}(x, t)$ is given by

$$
j_{\mu}=\frac{1}{2 \pi} \frac{1}{\sqrt{m_{\alpha} m^{\alpha}}} \epsilon^{\mu v} \epsilon^{\alpha \beta} m_{\alpha} \partial_{\nu} m_{\beta}, \quad \alpha, \beta=1,2,
$$

where $\mu, \nu=0,1$ correspond to the time and space components, respectively, and $\epsilon^{\mu \nu}, \epsilon^{\alpha \beta}$ are the two-index totally antisymmetric tensors in their respective indices. Note that $m_{3}$ does not enter the long-wavelength charge-response equation. If we parameterize $m_{1}=m \cos \theta, m_{2}=m \sin \theta$, then the response equation is simplified to

$$
\rho=\frac{1}{2 \pi} \partial_{x} \theta(x, t), \quad j=-\frac{1}{2 \pi} \partial_{t} \theta(x, t) .
$$




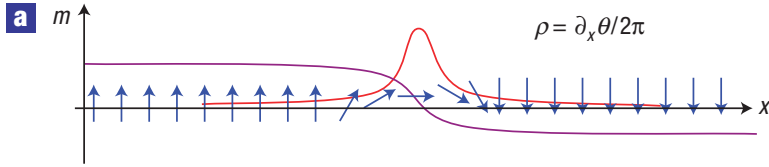

b

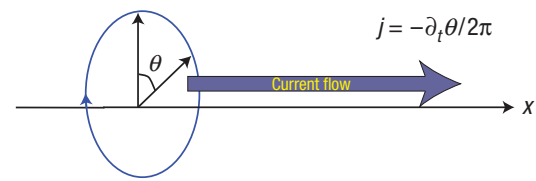

Figure 1 Illustrations of proposed phenomena. a, Schematic diagram of the half-charge domain wall. The blue arrows show a magnetic domain wall configuration and the purple line shows the mass kink. The red curve shows the charge density distribution. $\mathbf{b}$, Schematic diagram of the pumping induced by the rotation of the magnetic field. The blue circle with arrow shows the magnetic field rotation trajectory.

Such a response is 'topological' in the sense that the net charge $Q$ in a region $\left[x_{1}, x_{2}\right]$ at time $t$ depends only on the boundary values of $\theta(x, t)$, that is, $Q=\left[\theta\left(x_{2}, t\right)-\theta\left(x_{1}, t\right)\right] / 2 \pi$. In particular, a half-charge $\pm e / 2$ is carried by an antiphase domain wall of $\theta$, as shown in Fig. 1a (ref. 12). Similarly, the charge pumped by a purely time-dependent $\theta(t)$ field in a time interval $\left[t_{1}, t_{2}\right]$ is $\left.\Delta Q_{\text {pump }}\right|_{t_{2}} ^{t_{2}}=\left[\theta\left(t_{2}\right)-\theta\left(t_{1}\right)\right] / 2 \pi$. When $\theta$ is rotated from 0 to $2 \pi$ adiabatically, a quantized charge $e$ is pumped through the onedimensional system, as shown in Fig. 1b.

From the linear relation $m_{a}=t_{a i} B_{i}$, the angle $\theta$ can be determined for a given magnetic field $B_{i}$ as $\theta(x, t)=$ $\theta(\mathbf{B}(x, t))=\operatorname{Im} \log \left(\mathbf{t}_{1} \cdot \mathbf{B}(x, t)+i \mathbf{t}_{2} \cdot \mathbf{B}(x, t)\right)$, in which $\mathbf{t}_{1(2)}$ is the three-dimensional vector with components $t_{1(2) i}$, respectively. As $\theta(\mathbf{B})=\theta(-\mathbf{B})+\pi$, the charge localized on an antiphase magnetic domain wall of the magnetic field is always $\pm e / 2$. For the pumping effect, the winding number of $\theta(t)$ is given by the winding number of the $\mathbf{B}$ vector around the axis $\mathbf{t}_{1} \times \mathbf{t}_{2}$. The conditions for these effects to be observed are $k_{\mathrm{B}} T, \hbar \omega \ll E_{\mathrm{g}}$, where $k_{\mathrm{B}} T$ is the energy scale for temperature $T, \omega$ is the pumping frequency and $E_{\mathrm{g}}=\sqrt{\left(\mathbf{t}_{1} \cdot \mathbf{B}\right)^{2}+\left(\mathbf{t}_{2} \cdot \mathbf{B}\right)^{2}}$ is the energy gap of the helical edge state generated by the magnetic field.

The coefficients $t_{a i}$ for $\mathrm{HgTe} / \mathrm{CdTe}$ quantum wells can be obtained numerically by solving the four-band effective model given in ref. 6 with the addition of terms that result from bulk inversion asymmetry (see the Methods section). For a quantum well with thickness $d=70 \AA$ and an edge along the $y$ direction, we obtain $\mathbf{t}_{1}=(-0.3,0,0) \mathrm{meV} \mathrm{T}^{-1}$ and $\mathbf{t}_{2}=(0,-0.3,-3.1) \mathrm{meV} \mathrm{T}^{-1}$ (ref. 15). (Here and below the $z$ direction is the quantum well growth direction.) Thus, the gap induced by an in-plane field $B_{x}=1 \mathrm{~T}$ is $E_{\mathrm{g} x} \simeq 0.3 \mathrm{meV}$, whereas the perpendicular component $B_{z}=1 \mathrm{~T}$ produces a much larger gap $E_{\mathrm{g} z} \simeq 3.1 \mathrm{meV}$. (Such a large anisotropy between in-plane and perpendicular magnetic fields agrees well with the experimental observations in ref. 7 .) Consequently, the charge fractionalization effect can be observed at temperatures $T \ll 35 \mathrm{~K}$ on a domain wall with a perpendicular magnetic field, whereas the adiabatic pumping effect can only be observed at much lower temperatures $T \ll 3.5 \mathrm{~K}$ because it depends partially on the gap from in-plane fields.

Recently, a novel device has been developed to measure the charge of a confined region: the single-electron transistor ${ }^{16-18}$ (SET). This device can sensitively measure charges comparable to or even smaller than the electronic charge ${ }^{18,19}$ (see the Methods section). The fractional charge created by a magnetic domain wall on the QSH edge is confined in the region between the two

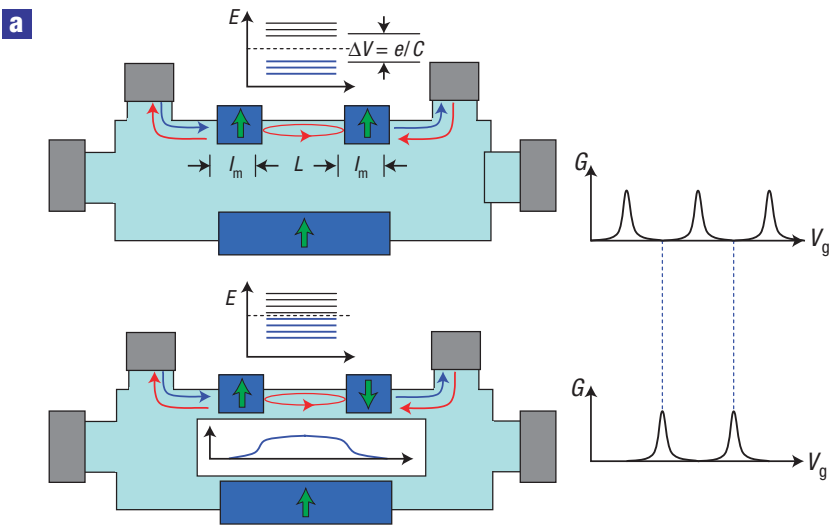

b

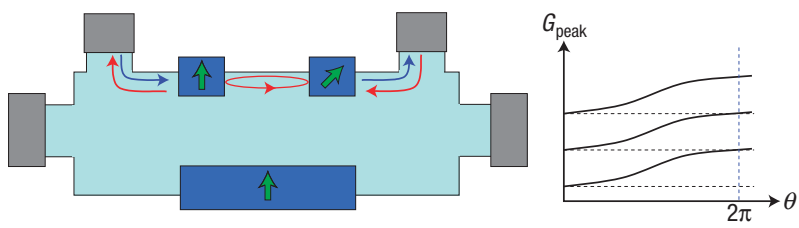

Figure 2 Experimental geometry of the SET device and proposed signature of static fractional charge. a, The SET device with parallel and antiparallel magnetic domains. The blue and grey rectangles are the magnetic domains and voltage probes, respectively. (In all of the diagrams, the magnetic domain on the bottom arm is always pinned, which is designed to block the transport of that arm so that the conductance of the SET device on the upper arm can be measured.) The conductance peak shift is shown on the right. The inset shows a schematic diagram of the bound-state wavefunction. $\mathbf{b}$, Schematic diagram of the conductance peak positions $G_{\text {peak }}$ being shifted by continuous rotation of the magnetic field. (Here and in Fig. 3, the actual direction of the rotating magnetic field should be in the plane perpendicular to the edge.)

magnetic domains separated by the wall. This confined charge can be measured by designing a magnetic SET experiment. A schematic diagram of such a device is shown in Fig. 2. Two magnetic islands can trap the electrons between them, just like a quantum wire trapped between two potential barriers ${ }^{17}$. In such a device, the conductance oscillations can be observed as in typical Coulomb-blockade measurements. The background charge in the confined region consists of two parts: $Q_{b}=Q_{c}+Q_{e}$, with $Q_{e}$ being the contribution of the lowest sub-band electrons and $Q_{c}$ being that of higher-energy bands and nuclei. When the field direction in one of the magnetic domains is switched, $Q_{c}$ remains invariant but $Q_{\mathrm{e}}$ will change by $e / 2$, which demonstrates the halfcharge associated to the antiphase domain wall. Consequently, if we use a top gate on the confined region and measure the conductance oscillations $G(V)$, there will be a half-period phase shift between the oscillation pattern of parallel and antiparallel magnetic domains, as shown in Fig. 2.

Experimentally, magnetic islands can be deposited on top of semiconductor heterostructures creating a hybrid ferromagnetsemiconductor device ${ }^{20,21}$. The magnetic islands can be polarized by magnetic field and locally switched using a coercive field and conventional magnetic-force microscopy techniques (see, for example, ref. 22). The quantum well will be locally exposed to the fringe fields of the ferromagnetic islands. To observe the conductance oscillations, several conditions should be satisfied by the magnetic field configuration which are outlined in the Methods section. 


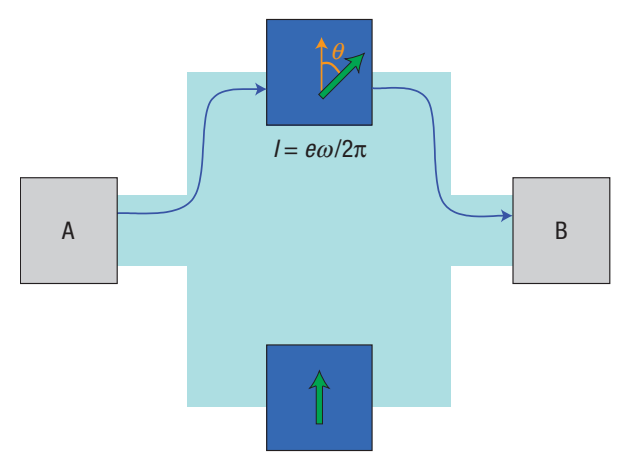

Figure 3 Experimental geometry for current generated from adiabatic pumping. Schematic diagram of the device for measuring the quantized charge current. A and $B$ are source and drain without an applied voltage bias between them.

So far, our focus has been on a magnetic domain wall structure that is externally imposed. However, it is also possible that the system could spontaneously generate such magnetic domain walls. In refs 8,9 , it was shown that two-particle backscattering interactions are allowed in the helical liquid. In the strong coupling limit, such a process can lead to a spontaneous breaking of timereversal symmetry, and spontaneous generation of a magnetic moment at the edge. This symmetry breaking is described by an Ising-like $Z_{2}$ order parameter, which at finite temperature in one dimension leads to a finite density of magnetic domain walls. Our work shows that such domain walls, which are the elementary excitations of the system, will carry fractional charge $\pm e / 2$.

We remark that the phenomenon of fractional charge associated with a magnetic domain wall is an example of 'electromagnetic duality' in one dimension. Here, a domain wall is a point-like object, and is dual to a point particle. In our particular case, a magnetic domain wall induces an electric point charge $(e / 2 \pi) \Delta \theta$. In three dimensions, a natural magnetic point singularity is a magnetic monopole, and Witten showed ${ }^{23}$ that it can induce an electric point charge $(e / 2 \pi) \theta$, where $\theta$ is the vacuum angle of quantum chromodynamics. The duality between a magnetic point charge and an electric point charge in the helical liquid of the QSH state has many other profound consequences which we shall demonstrate in future publications.

If the magnetic moment of one domain in the proposed SET device is rotated continuously by a full period while the other one remains static, the conductance peak position will shift relatively by a full period, as shown in Fig. 2 b. Such a shift of the conductance peaks shows the change of background charge by $e$ in the confined region, which is a consequence of the topological pumping effect. Another device to measure the pumping current directly is shown in Fig. 3. One strongly pinned and one easyplane magnetic island are deposited above one of each of the two arms of the device, respectively. When applying a small rotating external field with frequency $\omega$, the magnetization of the easyplane island will be rotated while the pinned one remains static. Consequently, a quantized charge current is pumped, given by the formula $I=e \omega / 2 \pi$ in the adiabatic approximation. To observe such an effect, the magnetic field-induced gap must be much larger than the temperature.

We have shown the feasibility of two striking topological effects in the QSH state. Using a single-electron transistor-like sensor, we proposed an experimental setting to create and observe the fractionally charged domain wall. Such topological phenomena, if observed, not only provide the first experimental realization of the fractional charge in one-dimensional systems, but also introduce a physical and operational definition of the two-dimensional topological (QSH) insulator. An (infinitesimal) magnetic field domain wall configuration can be used as a sensor to characterize two-dimensional insulators. If such a detection device induces a localized fractional charge response on the sample edge, then the system is defined to be a topological insulator. Such a definition is experimentally meaningful because it is based on the response of the system to some physical external field, and is completely analogous to the definition of the quantum Hall insulator as a system that produces a quantized Hall response to an external electric field.

\section{METHODS}

As shown in ref. 6, the low-energy physics of a $\mathrm{HgTe} / \mathrm{CdTe}$ quantum well is described by a $4 \times 4$ effective model, which can be obtained from a standard envelope function approach starting from the Kane mode ${ }^{24}$. Once the effective model $H(\mathbf{k})$ is obtained, the edge states can be obtained by diagonalizing the hamiltonian with a lattice regularization on a cylindrical geometry. Denoting $|k, \pm\rangle$ as the two counterpropagating edge states on the same physical boundary, the low-energy behaviour of these two channels is described by the one-dimensional hamiltonian (1). To leading order in magnetic field, the bulk effective hamiltonian can be expanded as $H(\mathbf{B}) \simeq H_{0}+H_{1}(\mathbf{B}) \equiv H_{0}+\hat{\mathbf{M}} \cdot \mathbf{B}+\hat{\mathbf{J}} \cdot \mathbf{A}$, where the last two terms represent the Zeeman coupling and orbital effect of the magnetic field, respectively. Only the perpendicular magnetic field $B_{z}$ contributes to $\mathbf{A}$. The matrix element of $H_{1}$ between the edge states $|k=0, \pm\rangle$ is $m_{\alpha \beta}(\mathbf{B})=\left\langle k=0, \alpha\left|H_{1}\right| k=0, \beta\right\rangle$, from which the coefficients $t_{a i}$ can be determined as $t_{a i}=(1 / 2) \operatorname{tr}\left[\sigma_{a}\left(\partial m / \partial B_{i}\right)_{\mathrm{B}=0}\right]$.

It should be noted that the perturbative calculation here is done for the two-dimensional bulk effective hamiltonian, although the resulting edge theory is still one-dimensional. Specifically, the two-dimensional bulk effects of the magnetic field are included in the orbital term $\hat{\mathbf{J}} \cdot \mathbf{A}$. In ref. 6 , the effective hamiltonian is block diagonal with the two counterpropagating edge states in different blocks, so that the current operator $\hat{\mathbf{J}}$ is also block diagonal and a perpendicular magnetic field cannot open a gap between edge states. However, the block-diagonal nature of the hamiltonian is not preserved when the bulk inversion asymmetry of the zinc-blende lattice is taken into account. This leads to terms $H_{\mathrm{BIA}}=\Delta\left(-\left|E_{1}+\right\rangle\left\langle H_{1}-|+| E_{1}-\right\rangle\left\langle H_{1}+\right|\right)+$ h.c. With such terms, the edge state gap induced by perpendicular magnetic field is non-zero. Owing to the non-trivial bulk topology, the orbital effect of a perpendicular magnetic field is quite strong, which makes a perpendicular field much more efficient than an in-plane field for opening the edge-state gap, even though the $\Delta$ term is small and usually subsidiary in other effects. More details concerning the perturbation procedure and the inclusion of bulk inversion breaking terms will be reserved for a future publication that deals with these specific issues.

The SET device uses the Coulomb blockade phenomenon to sensitively measure charge in a local region. When applying a gate voltage $V$ on top of a confined region (for example, quantum dot or wire) with capacitance $C$, the background charge induced by the gate screening is given by $Q_{b}=C V$. The Coulomb energy cost for an extra electron to enter the confined region has a period- $e$ dependence on the background charge $Q_{b}$, and vanishes at $Q_{\mathrm{b}}=(n+1 / 2) e$ with $n$ being an integer. Consequently, the $I-V$ curve of the confined region shows an oscillation with period $e / C$ and reaches its maximum at $V=(n+1 / 2) e / C$, where the average net charge of the confined region is a half-odd integer multiplied by the electron charge. For more details, see ref. 17.

For the fractional charge effect to be observed in the proposed SET device, the following conditions should be satisfied by the magnetic configuration in the hybrid semiconductor-ferromagnet device.

(1) The oscillation period $\Delta V=e / C$ should be much smaller than the bulk gap scale $V=E_{\mathrm{g}} / e$ so that several periods of oscillation can be observed before the gate voltage is so high that the bulk states are activated. This leads to the requirement $C \gg e^{2} / E_{\mathrm{g}}$.

(2) The ratio of the minimal conductance to the maximal conductance is estimated by

$$
G_{\min } / G_{\max } \simeq \exp \left[-\frac{e^{2} / 2 C}{k_{\mathrm{B}} T}\right]
$$


with $e^{2} / 2 C$ being the maximal charge activation gap. For the oscillations to be observable, this ratio should be reasonably smaller than 1 , which leads to the condition $e^{2} / 2 C \geq k_{\mathrm{B}} T$ or $C \leq e^{2} / 2 k_{\mathrm{B}} T$.

(3) The domain wall state trapped between the magnetic domains has an exponential tail as shown in Fig. 2. To make the conductance measurement, the size of the magnetic islands should be comparable to the exponential tail length $\xi_{\mathrm{M}} \simeq \hbar v_{\mathrm{F}} / E_{\mathrm{g}}$, with $E_{\mathrm{g}}$ being the magnetic-field-induced gap, so that the localized state is well confined, but tunnelling through the barrier is still strong enough to support observable transport.

The edge state trapped between magnetic domains has the linear size $\xi \times d \times L$, where $\xi \simeq \hbar v_{\mathrm{F}} / M$ is the penetration depth of the edge state, $d$ is the thickness of the quantum well and $L$ is the distance between the two magnetic regions. In the one-dimensional approximation $L \gg d$, $\xi$, we obtain the approximate form of the capacitance $C \simeq 4 \pi \epsilon_{0} L /\left[\log \left(L^{2} / d \xi\right)-2\right]$. For $d=70 \AA$, we find the condition $1 \mu \mathrm{m} \ll L \ll 100 \mu \mathrm{m}$. For a magnetic field $B_{z}=1 \mathrm{~T}$, the size of each magnetic island is $r \sim 120 \mathrm{~nm}$.

\section{Received 3 October 2007; accepted 14 February 2008; published 16 March 2008.}

\section{References}

1. Murakami, S., Nagaosa, N. \& Zhang, S. C. Dissipationless quantum spin current at room temperature. Science 301, 1348-1351 (2003).

2. Sinova, J. et al. Universal intrinsic spin Hall effect. Phys. Rev. Lett. 92, 126603 (2004).

3. Murakami, S., Nagaosa, N. \& Zhang, S. C. Spin-Hall insulator. Phys. Rev. Lett. 93, 156804 (2004).

4. Kane, C. L. \& Mele, E. J. Quantum spin Hall effect in graphene. Phys. Rev. Lett. 95, 226801 (2005).

5. Bernevig, B. A. \& Zhang, S. C. Quantum spin Hall effect. Phys. Rev. Lett. 96, 106802 (2006).

6. Bernevig, B. A., Hughes, T. L. \& Zhang, S. C. Quantum spin Hall effect and topological phase transition in HgTe quantum wells. Science 314, 1757-1761 (2006).

7. König, M. et al. Quantum spin Hall insulator state in HgTe quantum wells. Science 318, 766-770 (2007).

8. Wu, C., Bernevig, B. A. \& Zhang, S. C. Helical liquid and the edge of quantum spin Hall systems. Phys. Rev. Lett. 96, 106401 (2006).
9. Xu, C. \& Moore, J. E. Stability of the quantum spin Hall effect: Effects of interactions, disorder, and $Z_{2}$ topology. Phys. Rev. B 73, 045322 (2006).

10. Su, W. P., Schrieffer, J. R. \& Heeger, A. J. Solitons in polyacetylene. Phys. Rev. Lett. 42, 1698-1701 (1979).

11. Lee, D. H., Zhang, G. M. \& Xiang, T. Edge solitons of topological insulators and fractionalized quasiparticles in two dimensions. Phys. Rev. Lett. 99, 196805 (2007).

12. Jackiw, R. \& Rebbi, C. Solitons with fermion number 1/2. Phys. Rev. D 13, 3398-3409 (1976).

13. Thouless, D. J. Quantization of particle transport. Phys. Rev. B 27, 6083-6087 (1983).

14. Goldstone, J. \& Wilczek, F. Fractional quantum numbers on solitons. Phys. Rev. Lett. 47, 986-989 (1981).

15. Novik, E. G. et al. Band structure of semimagnetic $\mathrm{Hg}_{1-\gamma} \mathrm{Mn}_{\gamma}$ Te quantum wells. Phys. Rev. B 72, 035321 (2005).

16. Kastner, M. A., Kwasnick, R. F., Licini, J. C. \& Bishop, D. J. Conductance fluctuations near the localized-to-extended transition in narrow Si metal-oxide-semiconductor field-effect transistors. Phys. Rev. B 36, 8015-8031 (1987).

17. Kastner, M. A. The single-electron transistor. Rev. Mod. Phys. 64, 849-858 (1992).

18. Yoo, M. J. et al. Scanning single-electron transistor microscopy: Imaging individual charges. Science 276, 579-582 (1997)

19. Martin, J. et al. Localization of fractionally charged quasi-particles. Science 305 980-983 (2004).

20. Prinz, G. A. Hybrid ferromagnetic-semiconductor structure. Science 250, 1092-1097 (1990).

21. Halm, S. et al. Local spin manipulation in ferromagnet-semiconductor hybrids. Appl. Phys. Lett. 90 051916 (2007).

22. Kleiber, M. et al. Magnetization switching of submicrometer $\mathrm{CO}$ dots induced by a magnetic force microscope tip. Phys. Rev. B 58, 5563-5567 (1998).

23. Witten, E. Dyons of charge $e \theta / 2 \pi$. Phys. Lett. B 86, 283-287 (1979).

24. Kane, E. O. Band structure of InSb. J. Phys. Chem. Solids 1, 249-261 (1957).

\section{Acknowledgements}

We wish to thank B. A. Bernevig, H. Buhmann, X. Dai, C. L. Kane, M. Koenig and L. Molenkamp for insightful discussions. We acknowledge C.-X. Liu for sharing his unpublished numerical results. This work is supported by the NSF through the grants DMR-0342832, by the US Department of Energy, Office of Basic Energy Sciences under contract DE-AC03-76SF00515, and the Focus Center Research Program (FCRP) Center on Functional Engineered Nanoarchitectonics (FENA).

Correspondence and requests for materials should be addressed to S.-C.Z.

Reprints and permission information is available online at http://npg.nature.com/reprintsandpermissions/ 